\title{
Association Between Opioid and Benzodiazepine Use and Clinical Deterioration in Ward Patients
}

\author{
Patrick G. Lyons, MD¹, Ashley Snyder, $\mathrm{MPH}^{2}$, Sarah Sokol, PharmD³, Dana P. Edelson, MD, MS², \\ Babak Mokhlesi, MD, MSc${ }^{4}$, Matthew M. Churpek, MD, MPH, PhD**
}

\begin{abstract}
${ }^{1}$ Washington University School of Medicine, Department of Medicine, Division of Pulmonary and Critical Care Medicine, St. Louis, Missouri; ${ }^{2}$ The University of Chicago Medicine, Department of Medicine, Section of Hospital Medicine, Chicago, Illinois; ${ }^{3} T h e$ University of Chicago Medicine, Department of Pharmaceutical Services, Chicago, Illinois; ${ }^{4}$ The University of Chicago Medicine, Department of Medicine, Section of Pulmonary and Critical Care Medicine, Chicago, Illinois.
\end{abstract}

BACKGROUND: Opioids and benzodiazepines are frequently used in hospitals, but little is known about outcomes among ward patients receiving these medications.

OBJECTIVE: To determine the association between opioid and benzodiazepine administration and clinical deterioration.

DESIGN: Observational cohort study.

SETTING: 500-bed academic urban tertiary-care hospital.

PATIENTS: All adults hospitalized on the wards from November 2008 to January 2016 were included. Patients who were "comfort care" status, had tracheostomies, sickle-cell disease, and patients at risk for alcohol withdrawal or seizures were excluded.

MEASUREMENTS: The primary outcome was the composite of intensive care unit transfer or ward cardiac arrest. Discrete-time survival analysis was used to calculate the odds of this outcome during exposed time periods compared to unexposed time periods with respect to the medications of interest, with adjustment for patient demographics, comorbidities, severity of illness, and pain score.

RESULTS: In total, 120,518 admissions from 67,097 patients were included, with $67 \%$ of admissions involving opioids, and $21 \%$ involving benzodiazepines. After adjustment, each equivalent of $15 \mathrm{mg}$ oral morphine was associated with a $1.9 \%$ increase in the odds of the primary outcome within 6 hours (odds ratio [OR], 1.019; 95\% confidence interval [Cl], 1.013-1.026; $P<0.001$ ), and each $1 \mathrm{mg}$ oral lorazepam equivalent was associated with a $29 \%$ increase in the odds of the composite outcome within 6 hours (OR, 1.29; Cl, 1.161.45; $P<0.001)$.

CONCLUSION: Among ward patients, opioids were associated with increased risk for clinical deterioration in the 6 hours after administration. Benzodiazepines were associated with even higher risk. These results have implications for ward-monitoring strategies. Journal of Hospital Medicine 2017;12:428-434. (c) 2017 Society of Hospital Medicine
Chronic opioid and benzodiazepine use is common and increasing. ${ }^{1-5}$ Outpatient use of these medications has been associated with hospital readmission and death, ${ }^{6-12}$ with concurrent use associated with particularly increased risk. ${ }^{13,14}$ Less is known about outcomes for hospitalized patients receiving these medications.

More than half of hospital inpatients in the United States receive opioids, ${ }^{15}$ many of which are new prescriptions rather than continuation of chronic therapy. ${ }^{16,17}$ Less is known about inpatient benzodiazepine administration, but the prevalence may exceed $10 \%$ among elderly populations. ${ }^{18}$ Hospitalized patients often have comorbidities or physiological disturbances that might increase their risk related to use of these medications. Opioids can cause central and obstructive sleep apneas, ${ }^{19-21}$ and benzodiazepines contribute to respiratory depression and airway relaxation. ${ }^{22}$ Benzodiaz-

*Address for correspondence and reprint requests: Matthew M. Churpek, $\mathrm{MD}, \mathrm{MPH}, \mathrm{PhD}$, University of Chicago, Section of Pulmonary and Critical Care, 5841 S. Maryland Avenue, MC 6076, Chicago, IL 60637; Telephone: 773-7021092; Fax: 773-702-6500; E-mail: matthew.churpek@uchospitals.edu

Additional Supporting Information may be found in the online version of this article.

Received: November 1, 2016; Revised: February 10, 2017; Accepted: February 28, 2017

2017 Society of Hospital Medicine DOI 10.12788/jhm.2749 epines also impair psychomotor function and recall, ${ }^{23}$ which could mediate the recognized risk for delirium and falls in the hospital. ${ }^{24,25}$ These findings suggest pathways by which these medications might contribute to clinical deterioration.

Most studies in hospitalized patients have been limited to specific populations ${ }^{15,26-28}$ and have not explicitly controlled for severity of illness over time. It remains unclear whether associations identified within particular groups of patients hold true for the broader population of general ward inpatients. Therefore, we aimed to determine the independent association between opioid and benzodiazepine administration and clinical deterioration in ward patients.

\section{MATERIALS AND METHODS}

\section{Setting and Study Population}

We performed an observational cohort study at a 500-bed urban academic hospital. Data were obtained from all adults hospitalized on the wards between November 1, 2008, and January 21, 2016. The study protocol was approved by the University of Chicago Institutional Review Board (IRB\#15-0195).

\section{Data Collection}

The study utilized de-identified data from the electronic health record (EHR; Epic Systems Corporation, Verona, 


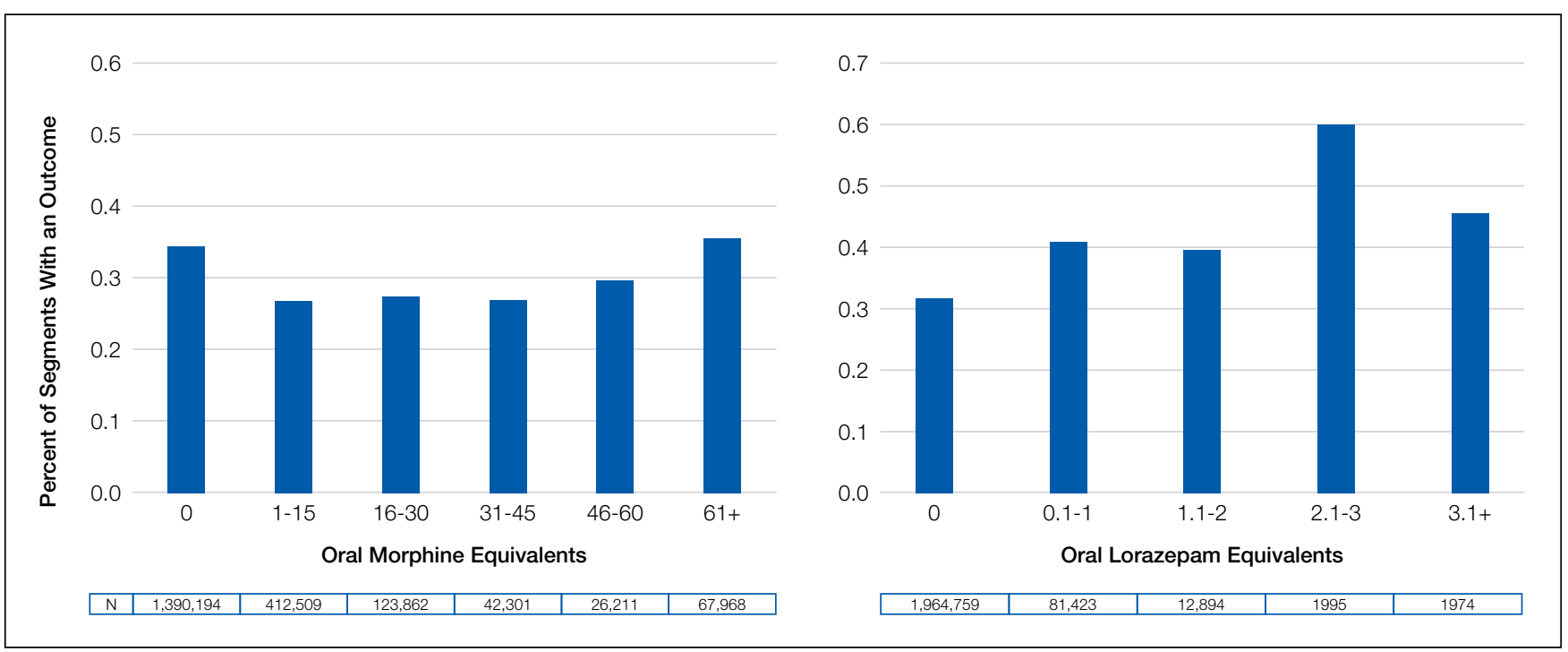

FIG. Unadjusted frequency of composite outcome stratified by medication dose. ${ }^{a}$

aWard cardiac arrest or intensive care unit transfer.

NOTE: N reflects the number of 6-hr ward segments associated with each dosing range, and the Y-axis shows the percentage of 6-hr segments in which an outcome occurred.

Wisconsin) and administrative databases collected by the University of Chicago Clinical Research Data Warehouse. Patient age, sex, race, body mass index (BMI), and ward admission source (ie, emergency department (ED), transferred from the intensive care unit (ICU), or directly admitted to the wards) were collected. International Classification of Diseases, $9^{\text {th }}$ Revision, Clinical Modification (ICD-9-CM) codes were used to identify Elixhauser Comorbidity Index categories. ${ }^{29,30}$ Because patients with similar diagnoses (eg, active cancer) are cohorted within particular areas in our hospital, we obtained the ward unit for all patients. Patients who underwent surgery were identified using the hospital's admission-transfer-discharge database.

To determine severity of illness, routinely collected vital signs and laboratory values were utilized to calculate the electronic cardiac arrest risk triage (eCART) score, an accurate risk score we previously developed and validated for predicting adverse events among ward patients. ${ }^{31}$ If any vital sign or laboratory value was missing, the next available measurement was carried forward. If any value remained missing after this change, the median value for that location (ie, wards, ICU, or ED) was imputed..$^{32,33}$ Additionally, patient-reported pain scores at the time of opioid administration were extracted from nursing flowsheets. If no pain score was present at the time of opioid administration, the patient's previous score was carried forward.

We excluded patients with sickle-cell disease or seizure history and admissions with diagnoses of alcohol withdrawal from the analysis, because these diagnoses were expected to be associated with different medication administration practices compared to other inpatients. We also excluded patients with a tracheostomy because we expected their respiratory monitoring to differ from the other patients in our cohort. Finally, because ward deaths resulting from a com- fort care scenario often involve opioids and/or benzodiazepines, ward segments involving comfort care deaths (defined as death without attempted resuscitation) were excluded from the analysis (Supplemental Figure 1). Patients with sickle-cell disease were identified using ICD-9 codes, and encounters during which a seizure may have occurred were identified using a combination of ICD-9 codes and receipt of anti-epileptic medication (Supplemental Table 1). Patients at risk for alcohol withdrawal were identified by the presence of any Clinical Institute Withdrawal Assessment for Alcohol score within nursing flowsheets, and patients with tracheostomies were identified using documentation of ventilator support within their first 12 hours on the wards. In addition to these exclusion criteria, patients with obstructive sleep apnea (OSA) were identified by the following ICD-9 codes: $278.03,327.23,780.51,780.53$, and 780.57.

\section{Medications}

Ward administrations of opioids and benzodiazepines-dose, route, and administration time-were collected from the EHR. We excluded all administrations in nonward locations such as the ED, ICU, operating room, or procedure suite. Additionally, because patients emergently intubated may receive sedative and analgesic medications to facilitate intubation, and because patients experiencing cardiac arrest are frequently intubated periresuscitation, we a priori excluded all administrations within 15 minutes of a ward cardiac arrest or an intubation.

For consistent comparisons, opioid doses were converted to oral morphine equivalents $\mathrm{s}^{34}$ and adjusted by a factor of 15 to reflect the smallest routinely available oral morphine tablet in our hospital (Supplemental Table 2). Benzodiazepine doses were converted to oral lorazepam equivalents (Supplemental Table 2). ${ }^{34}$ Thus, the independent variables were oral 
TABLE 1. Characteristics of Patient Admissions During Which Opioids and Benzodiazepines Were and Were Not Administered

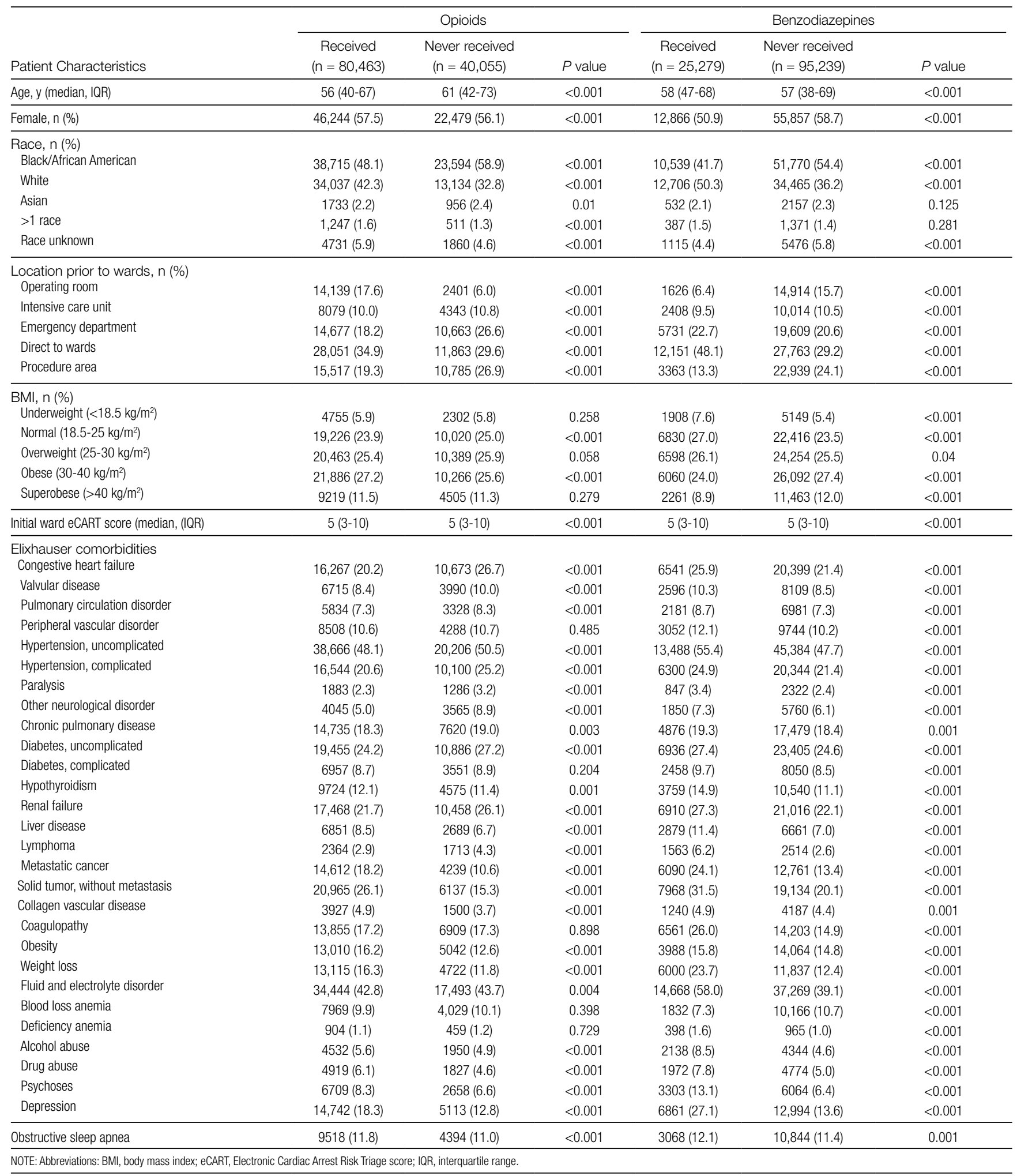

morphine or lorazepam equivalents administered within each 6-hour window. We a priori presumed opioid doses greater than the $99^{\text {th }}$ percentile $(1200 \mathrm{mg})$ or benzodiazepine doses greater than $10 \mathrm{mg}$ oral lorazepam equivalents within a 6-hour window to be erroneous entries, and replaced these outlier values with the median value for each medication category. 


\section{Outcomes}

The primary outcome was the composite of ICU transfer or cardiac arrest (loss of pulse with attempted resuscitation) on the wards, with individual outcomes investigated secondarily. An ICU transfer (patient movement from a ward directly to the ICU) was identified using the hospital's admission-transfer-discharge database. Cardiac arrests were identified using a prospectively validated quality improvement database. ${ }^{35}$

Because deaths on the wards resulted either from cardiac arrest or from a comfort care scenario, mortality was not studied as an outcome.

\section{Statistical Analysis}

Patient characteristics were compared using Student t tests, Wilcoxon rank sum tests, and chi-squared statistics, as appropriate. Unadjusted and adjusted models were created using discrete-time survival analysis, ${ }^{36-39}$ which involved dividing time into discrete 6-hour intervals and employing the predictor variables chronologically closest to the beginning of each time window to forecast whether the outcome occurred within each interval. Predictor variables in the adjusted model included patient characteristics (age, sex, BMI, and Elixhauser Agency for Healthcare Research and Quality-Web comorbidities $^{30}$ [a priori excluding comorbidities recorded for fewer than 1000 admissions from the model]), ward unit, surgical status, prior ICU admission during the hospitalization, cumulative opioid or benzodiazepine dose during the previous 24 hours, and severity of illness (measured by eCART score). The adjusted model for opioids also included the patient's pain score. Age, eCART score, and pain score were entered linearly while race, BMI (underweight, less than $18.5 \mathrm{~kg} / \mathrm{m}^{2}$; normal, 18.5-24.9 kg/m²; overweight, 25.0-29.9 kg/m²; obese, $30-39.9 \mathrm{~kg} / \mathrm{m}^{2}$; and severely obese, $40 \mathrm{mg} / \mathrm{m}^{2}$ or greater), and ward unit were modeled as categorical variables.

Since repeat hospitalization could confound the results of our study, we performed a sensitivity analysis including only 1 randomly selected hospital admission per patient. We also performed a sensitivity analysis including receipt of both opioids and benzodiazepines, and an interaction term within each ward segment, as well as an analysis in which zolpidem-the most commonly administered nonbenzodiazepine hypnotic medication in our hospital—was included along with both opioids and benzodiazepines. Finally, we performed a sensitivity analysis replacing missing pain scores with imputed values ranging from 0 to the median ward pain score.

We also performed subgroup analyses of adjusted models across age quartiles and for each BMI category, as well as for surgical status, OSA status, gender, time of medication administration, and route of administration (intravenous vs. oral). We also performed an analysis across pain score severity ${ }^{40}$ to determine whether these medications produce differential effects at various levels of pain.

All tests of significance used a 2 -sided $P$ value less than 0.05. Statistical analyses were completed using Stata version 14.1 (StataCorp, LLC, College Station, Texas).

\section{RESULTS}

\section{Patient Characteristics}

A total of 144,895 admissions, from 75,369 patients, had ward vital signs or laboratory values documented during the study period. Ward segments from 634 admissions were excluded due to comfort care status, which resulted in exclusion of 479 complete patient admissions. Additionally, 139 patients with tracheostomies were excluded. Furthermore, 2934 patient admissions with a sickle-cell diagnosis were excluded, of which $95 \%(n=2791)$ received an opioid and $11 \%(n=310)$ received a benzodiazepine. Another 14,029 admissions associated with seizures, 6134 admissions involving alcohol withdrawal, and 1332 with both were excluded, of which $66 \%(n=14,174)$ received an opioid and $35 \%$ $(\mathrm{n}=7504)$ received a benzodiazepine. After exclusions, 120,518 admissions were included in the final analysis, with $67 \%(n=80,463)$ associated with at least 1 administration of an opioid and $21 \%(n=25,279)$ associated with at least 1 benzodiazepine administration.

In total, there were 672,851 intervals when an opioid was administered during the study, with a median dose of 12 mg oral morphine equivalents (interquartile range, 8-30). Of these, 21,634 doses were replaced due to outlier status outside the $99^{\text {th }}$ percentile. Patients receiving opioids were younger (median age 56 vs 61 years), less likely to be African American (48\% vs 59\%), more likely to have undergone surgery (18\% vs 6\%), and less likely to have most noncancer medical comorbidities than those who never received an opioid (all $P<0.001$ ) (Table 1 ).

Additionally, there were a total of 98,286 6-hour intervals in which a benzodiazepine was administered in the study, with a median dose of $1 \mathrm{mg}$ oral lorazepam (interquartile range, 0.5-1). A total of 790 doses of benzodiazepines (less than $1 \%$ ) were replaced due to outlier status. Patients who received benzodiazepines were more likely to be male $(49 \%$ vs. $41 \%$ ), less likely to be African-American, less likely to be obese or morbidly obese ( $33 \%$ vs. $39 \%$ ), and more likely to have medical comorbidities compared to patients who never received a benzodiazepine (all $P<0.001$ ) (Table 1 ).

The eCART scores were similar between all patient groups. The frequency of missing variables differed by data type, with vital signs rarely missing (all less than $1.1 \%$ except AVPU [10\%]), followed by hematology labs (8\%-9\%), electrolytes and renal function results (12\%-15\%), and hepatic function tests (40\%-45\%). In addition to imputed data for missing vital signs and laboratory values, our model omitted human immunodeficiency virus/acquired immune deficiency syndrome and peptic ulcer disease from the adjusted models on the basis of fewer than 1000 admissions with these diagnoses listed.

\section{Patient Outcomes}

The incidence of the composite outcome was higher in admissions with at least 1 opioid medication than those without an opioid ( $7 \%$ vs. $4 \%, P<0.001$ ), and in admissions with at least 1 dose of benzodiazepines compared to those without 
TABLE 2. Unadjusted Ward Outcome Rates for Patient Admissions With and Without Opioid or Benzodiazepine Administration

\begin{tabular}{|c|c|c|c|c|c|c|}
\hline \multirow[b]{2}{*}{ Outcomes, n (\%) } & \multicolumn{3}{|c|}{ Opioids } & \multicolumn{3}{|c|}{ Benzodiazepines } \\
\hline & $\begin{array}{c}\text { Received } \\
(\mathrm{n}=80,463)\end{array}$ & $\begin{array}{l}\text { Never received } \\
(n=40,055)\end{array}$ & $P$ value & $\begin{array}{c}\text { Received } \\
(n=25,279)\end{array}$ & $\begin{array}{c}\text { Never received } \\
(n=95,239)\end{array}$ & $P$ value \\
\hline ICU transfer & 5177 (6) & 1399 (4) & $<0.001$ & 2708 (11) & 3868 (4) & $<0.001$ \\
\hline Ward cardiac arrest & $174(0.2)$ & $70(0.2)$ & 0.135 & $87(0.3)$ & $157(0.2)$ & $<0.001$ \\
\hline
\end{tabular}

TABLE 3. Adjusted Odds of Clinical Deterioration Outcomes Within Six Hours of Receiving an Opioid or Benzodiazepine ${ }^{a}$

\begin{tabular}{|c|c|c|c|c|}
\hline \multirow[b]{2}{*}{ Outcome } & \multicolumn{2}{|c|}{ Opioids } & \multicolumn{2}{|c|}{ Benzodiazepines } \\
\hline & OR (95\% Cl) & $P$ value & OR $(95 \% \mathrm{Cl})$ & $P$ value \\
\hline Composite & $1.019(1.013-1.026)$ & $<0.001$ & $1.29(1.16-1.45)$ & $<0.001$ \\
\hline ICU transfer & $1.019(1.013-1.026)$ & $<0.001$ & $1.29(1.14-1.43)$ & $<0.001$ \\
\hline Ward cardiac arrest & $1.020(0.985-1.057)$ & 0.26 & $2.36(1.43-3.90)$ & 0.001 \\
\hline
\end{tabular}

aAdjustment includes patient characteristics, ward unit, surgical status, prior ICU admission, 24-hour cumulative opioid/benzodiazepine dose, and eCART score.

NOTE: Odds ratios reflect the change in odds associated with the equivalent of $15 \mathrm{mg}$ oral morphine or $1 \mathrm{mg}$ oral lorazepam. Abbreviations: eCART, Electronic Cardiac Arrest Risk Triage score; Cl, confidence interval; ICU, intensive care unit; $\mathrm{OR}$, odds ratio.

a benzodiazepine ( $11 \%$ vs. $4 \%, P<0.001$ ) (Table 2 ).

Within 6-hour segments, increasing doses of opioids were associated with an initial decrease in the frequency of the composite outcome followed by a dose-related increase in the frequency of the composite outcome with morphine equivalents greater than $45 \mathrm{mg}$. By contrast, the frequency of the composite outcome increased with additional benzodiazepine equivalents (Figure).

In the adjusted model, opioid administration was associated with increased risk for the composite outcome (Table 3) in a dose-dependent fashion, with each $15 \mathrm{mg}$ oral morphine equivalent associated with a $1.9 \%$ increase in the odds of ICU transfer or cardiac arrest within the subsequent 6-hour time interval (odds ratio [OR], 1.019; 95\% confidence interval $[\mathrm{CI}], 1.013-1.026 ; \mathrm{P}<0.001)$.

Similarly, benzodiazepine administration was also associated with increased adjusted risk for the composite outcome within 6 hours in a dose-dependent manner. Each $1 \mathrm{mg}$ oral lorazepam equivalent was associated with a $29 \%$ increase in the odds of ward cardiac arrest or ICU transfer (OR, 1.29; 95\% CI, 1.16-1.44; $P<0.001$ ) (Table 3).

\section{Sensitivity Analyses}

A sensitivity analysis including 1 randomly selected hospitalization per patient involved 67,097 admissions and found results similar to the primary analysis, with each $15 \mathrm{mg}$ oral morphine equivalent associated with a $1.9 \%$ increase in the odds of the composite outcome (OR, 1.019; 95\% CI, 1.0111.028; $P<0.001)$ and each $1 \mathrm{mg}$ oral lorazepam equivalent associated with a $41 \%$ increase in the odds of the composite outcome (OR, 1.41; 95\% CI, 1.21-1.65; P<0.001). Inclusion of both opioids and benzodiazepines in the adjusted model again yielded results similar to the main analysis for both opioids (OR, 1.020; 95\% CI, 1.013-1.026; P<0.001) and benzodiazepines (OR, 1.35; 95\% CI, 1.18-1.54; $P<0.001$ ), without a significant interaction detected $(P=0.09)$. These results were unchanged with the addition of zolpidem to the model as an additional potential confounder, and zolpidem did not increase the risk of the study outcomes $(P=0.2)$.

A final sensitivity analysis for the opioid model involved replacing missing pain scores with imputed values ranging from 0 to the median ward score, which was 5 . The results of these analyses did not differ from the primary model and were consistent regardless of imputation value $(\mathrm{OR}, 1.018$; 95\% CI, 1.012-1.023; P < 0.001).

\section{Subgroup Analyses}

Analyses of opioid administration by subgroup (sex, age quartiles, BMI categories, OSA diagnosis, surgical status, daytime/nighttime medication administration, IV/PO administration, and pain severity) yielded similar results to the overall analysis (Supplemental Figure 2). Subgroup analysis of patients receiving benzodiazepines revealed similarly increased adjusted odds of the composite outcome across strata of gender, BMI, surgical status, and medication administration time (Supplemental Figure 3). Notably, patients older than 70 years who received a benzodiazepine were at $64 \%$ increased odds of the composite outcome (OR, 1.64; 95\% CI, $1.30-2.08$ ), compared to $2 \%$ to $38 \%$ increased risk for patients under 70 years. Finally, IV doses of benzodiazepines were associated with $48 \%$ increased odds for deterioration (OR, 1.48; 95\% CI, 1.18-1.84; $P=0.001$ ), compared to a nonsignificant $14 \%$ increase in the odds for PO doses (OR, $1.14 ; 95 \%$ CI, 0.99-1.31; $P=0.066$ ). 


\section{DISCUSSION}

In a large, single-center, observational study of ward inpatients, we found that opioid use was associated with a small but significant increased risk for clinical deterioration on the wards, with every $15 \mathrm{mg}$ oral morphine equivalent increasing the odds of ICU transfer or cardiac arrest in the next 6 hours by $1.9 \%$. Benzodiazepines were associated with a much higher risk: each equivalent of $1 \mathrm{mg}$ of oral lorazepam increased the odds of ICU transfer or cardiac arrest by almost 30\%. These results have important implications for care at the bedside of hospitalized ward patients and suggest the need for closer monitoring after receipt of these medications, particularly benzodiazepines.

Previous work has described negative effects of opioid medications among select inpatient populations. In surgical patients, opioids have been associated with hospital readmission, increased length of stay, and hospital mortality. ${ }^{26,28}$ More recently, Herzig et al. ${ }^{15}$ found more adverse events in nonsurgical ward patients within the hospitals prescribing opioids the most frequently. These studies may have been limited by the populations studied and the inability to control for confounders such as severity of illness and pain score. Our study expands these findings to a more generalizable population and shows that even after adjustment for potential confounders, such as severity of illness, pain score, and medication dose, opioids are associated with increased shortterm risk of clinical deterioration.

By contrast, few studies have characterized the risks associated with benzodiazepine use among ward inpatients. Recently, Overdyk et al. ${ }^{27}$ found that inpatient use of opioids and sedatives was associated with increased risk for cardiac arrest and hospital death. However, this study included ICU patients, which may confound the results, as ICU patients often receive high doses of opioids or benzodiazepines to facilitate mechanical ventilation or other invasive procedures, while also having a particularly high risk of adverse outcomes like cardiac arrest and inhospital death.

Several mechanisms may explain the magnitude of effect seen with regard to benzodiazepines. First, benzodiazepines may directly produce clinical deterioration by decreased respiratory drive, diminished airway tone, or hemodynamic decompensation. It is possible that the broad spectrum of cardiorespiratory side effects of benzodiazepines-and potential unpredictability of these effects-increases the difficulty of observation and management for patients receiving them. This difficulty may be compounded with intravenous administration of benzodiazepines, which was associated with a higher risk for deterioration than oral doses in our cohort. Alternatively, benzodiazepines may contribute to clinical decompensation by masking signs of deterioration such as encephalopathy or vital sign instability like tachycardia or tachypnea that may be mistaken as anxiety. Notably, while our hospital has a nursing-driven protocol for monitoring patients receiving opioids (in which pain is serially assessed, leading to additional bedside observation), we do not have protocols for ward patients receiving benzodiazepines. Finally, although we found that orders for opioids and benzodiazepines were more common in white patients than African American patients, this finding may be due to differences in the types or number of medical comorbidities experienced by these patients.

Our study has several strengths, including the large number of admissions we included. Additionally, we included a broad range of medical and surgical ward admissions, which should increase the generalizability of our results. Further, our rates of ICU transfer are in line with data reported from other groups, ${ }^{41,42}$ which again may add to the generalizability of our findings. We also addressed many potential confounders by including patient characteristics, individual ward units, and (for opioids) pain score in our model, and by controlling for severity of illness with the eCART score, an accurate predictor of ICU transfer and ward cardiac arrest within our population. ${ }^{32,37}$ Finally, our robust methodology allowed us to include acute and cumulative medication doses, as well as time, in the model. By performing a discrete-time survival analysis, we were able to evaluate receipt of opioids and benzodiazepines-as well as risk for clinical deteriorationlongitudinally, lending strength to our results.

Limitations of our study include its single-center cohort, which may reduce generalizability to other populations. Additionally, because we could not validate the accuracy of-or adherence to-outpatient medication lists, we were unable to identify chronic opioid or benzodiazepine users by these lists. However, patients chronically taking opioids or benzodiazepines would likely receive doses each hospital day; by including 24-hour cumulative doses in our model, we attempted to adjust for some portion of their chronic use. Also, because evaluation of delirium was not objectively recorded in our dataset, we were unable to evaluate the relationship between receipt of these medications and development of delirium, which is an important outcome for hospitalized patients. Finally, neither the diagnoses for which these medications were prescribed, nor the reason for ICU transfer, were present in our dataset, which leaves open the possibility of unmeasured confounding.

\section{CONCLUSION}

After adjustment for important confounders including severity of illness, medication dose, and time, opioids were associated with a slight increase in clinical deterioration on the wards, while benzodiazepines were associated with a much larger risk for deterioration. This finding raises concern about the safety of benzodiazepine use among ward patients and suggests that increased monitoring of patients receiving these medications may be warranted.

\section{Acknowledgment}

The authors thank Nicole Twu for administrative support.

Disclosure: Drs. Churpek and Edelson have a patent pending (ARCD. P0535US.P2) for risk stratification algorithms for hospitalized patients. Dr. Churpek is supported 
by a career development award from the National Heart, Lung, and Blood Institute (K08 HL121080). Dr. Churpek has received honoraria from Chest for invited speaking engagements. In addition, Dr. Edelson has received research support from Philips Healthcare (Andover, Massachusetts), research support from the American Heart Association (Dallas, Texas) and Laerdal Medical (Stavanger, Norway), and research support from Early Sense (Tel Aviv, Israel). She has ownership interest in Quant HC

\section{References}

1. Substance Abuse and Mental Health Services Administration. Results from the 2013 National Survey on Drug Use and Health: Summary of National Findings. Rockville, MD: Substance Abuse and Mental Health Services Administration; 2014.

2. Bachhuber MA, Hennessy S, Cunningham CO, Starrels JL. Increasing benzodiazepine prescriptions and overdose mortality in the United States, 1996-2013. Am J Public Health. 2016;106(4):686-688.

3. Parsells Kelly J, Cook SF, Kaufman DW, Anderson T, Rosenberg L, Mitchell AA. Prevalence and characteristics of opioid use in the US adult population. Pain. 2008;138(3):507-513.

4. Olfson M, King M, Schoenbaum M. Benzodiazepine use in the United States. JAMA Psychiatry. 2015;72(2):136-142.

5. Hwang CS, Kang EM, Kornegay CJ, Staffa JA, Jones CM, McAninch JK. Trends in the concomitant prescribing of opioids and benzodiazepines, 2002-2014. Am J Prev Med. 2016;51(2):151-160.

6. Bohnert AS, Valenstein M, Bair MJ, et al. Association between opioid prescribing patterns and opioid overdose-related deaths. JAMA. 2011;305(13):1315-1321.

7. Dart RC, Surratt HL, Cicero TJ, et al. Trends in opioid analgesic abuse and mortality in the United States. N Engl J Med. 2015;372(3):241-248.

8. Centers for Disease Control and Prevention (CDC). Vital signs: overdoses of prescription opioid pain relievers---United States, 1999--2008. MMWR Morb Mortal Wkly Rep. 2011;60(43):1487-1492.

9. Lan TY, Zeng YF, Tang GJ, et al. The use of hypnotics and mortality - a population-based retrospective cohort study. PLoS One. 2015;10(12):e0145271.

10. Mosher HJ, Jiang L, Vaughan Sarrazin MS, Cram P, Kaboli P, Vander Weg MW Prevalence and characteristics of hospitalized adults on chronic opioid therapy: prior opioid use among veterans. J Hosp Med. 2014;9(2):82-87.

11. Palmaro A, Dupouy J, Lapeyre-Mestre M. Benzodiazepines and risk of death: results from two large cohort studies in France and UK. Eur Neuropsychopharmacol. 2015;25(10):1566-1577.

12. Parsaik AK, Mascarenhas SS, Khosh-Chashm D, et al. Mortality associated with anxiolytic and hypnotic drugs-a systematic review and meta-analysis. Aust N Z J Psychiatry. 2016;50(6):520-533.

13. Park TW, Saitz R, Ganoczy D, Ilgen MA, Bohnert AS. Benzodiazepine prescribing patterns and deaths from drug overdose among US veterans receiving opioid analgesics: case-cohort study. BMJ. 2015;350:h2698.

14. Jones CM, McAninch JK. Emergency department visits and overdose deaths from combined use of opioids and benzodiazepines. Am J Prev Med. 2015;49(4):493-501.

15. Herzig SJ, Rothberg MB, Cheung M, Ngo LH, Marcantonio ER. Opioid utilization and opioid-related adverse events in nonsurgical patients in US hospitals. J Hosp Med. 2014;9(2):73-81

16. Jena AB, Goldman D, Karaca-Mandic P. Hospital prescribing of opioids to Medicare beneficiaries. JAMA Intern Med. 2016;176(7):990-997.

17. Calcaterra SL, Yamashita TE, Min SJ, Keniston A, Frank JW, Binswanger IA Opioid prescribing at hospital discharge contributes to chronic opioid use. J Gen Intern Med. 2016;31(5):478-485.

18. Garrido MM, Prigerson HG, Penrod JD, Jones SC, Boockvar KS. Benzodiazepine and sedative-hypnotic use among older seriously ill veterans: choosing wisely? Clin Ther. 2014;36(11):1547-1554.

19. Doufas AG, Tian L, Padrez KA, et al. Experimental pain and opioid analgesia in volunteers at high risk for obstructive sleep apnea. PloS One. 2013;8(1):e54807.

20. Gislason T, Almqvist M, Boman G, Lindholm CE, Terenius L. Increased CSF opioid activity in sleep apnea syndrome. Regression after successful treatment. Chest. 1989;96(2):250-254.

21. Van Ryswyk E, Antic N. Opioids and sleep disordered breathing. Chest. 2016;150(4):934-944.

22. Koga Y, Sato S, Sodeyama N, et al. Comparison of the relaxant effects of diaz-
(Chicago, Illinois), which is developing products for risk stratification of hospitalized patients. Dr. Mokhlesi is supported by National Institutes of Health grant R01HL119161. Dr. Mokhlesi has served as a consultant to Philips/Respironics and has received research support from Philips/Respironics. Preliminary versions of these data were presented as a poster presentation at the 2016 meeting of the American Thoracic Society, May 17, 2016; San Francisco, California.

epam, flunitrazepam and midazolam on airway smooth muscle. $\mathrm{Br} J$ Anaesth. 1992;69(1):65-69.

23. Pomara N, Lee SH, Bruno D, et al. Adverse performance effects of acute lorazepam administration in elderly long-term users: pharmacokinetic and clinical predictors. Prog Neuropsychopharmacol Biol Psychiatry. 2015;56:129-135.

24. Pandharipande $P$, Shintani A, Peterson J, et al. Lorazepam is an independent risk factor for transitioning to delirium in intensive care unit patients. Anesthesiology. 2006;104(1):21-26.

25. O'Neil CA, Krauss MJ, Bettale J, et al. Medications and patient characteristics associated with falling in the hospital. J Patient Saf. 2015 (epub ahead of print).

26. Kessler ER, Shah M, K Gruschkus S, Raju A. Cost and quality implications of opioid-based postsurgical pain control using administrative claims data from a large health system: opioid-related adverse events and their impact on clinical and economic outcomes. Pharmacotherapy. 2013;33(4):383-391.

27. Overdyk FJ, Dowling O, Marino J, et al. Association of opioids and sedatives with increased risk of in-hospital cardiopulmonary arrest from an administrative database. PLoS One. 2016;11(2):e0150214.

28. Minkowitz HS, Gruschkus SK, Shah M, Raju A. Adverse drug events among patients receiving postsurgical opioids in a large health system: risk factors and outcomes. Am J Health Syst Pharm. 2014;71(18):1556-1565.

29. Elixhauser A, Steiner C, Harris DR, Coffey RM. Comorbidity measures for use with administrative data. Med Care. 1998;36(1):8-27.

30. Quan H, Sundararajan V, Halfon P, et al. Coding algorithms for defining comorbidities in ICD-9-CM and ICD-10 administrative data. Med Care. 2005;43(11):1130-1139.

31. Churpek MM, Yuen TC, Winslow C, et al. Multicenter development and validation of a risk stratification tool for ward patients. Am J Respir Crit Care Med. 2014;190(6):649-655

32. Knaus WA, Wagner DP, Draper EA, Z et al. The APACHE III prognostic system. Risk prediction of hospital mortality for critically ill hospitalized adults. Chest. 1991;100(6):1619-1636.

33. van den Boogaard M, Pickkers P, Slooter AJC, et al. Development and validation of PRE-DELIRIC (PREdiction of DELIRium in ICu patients) delirium prediction model for intensive care patients: observational multicentre study. BMJ. 2012;344:e420.

34. Clinical calculators. ClinCalc.com. http://www.clincalc.com. Accessed February 21, 2016.

35. Churpek MM, Yuen TC, Huber MT, Park SY, Hall JB, Edelson DP. Predicting cardiac arrest on the wards: a nested case-control study. Chest. 2012;141(5): 1170-1176.

36. Churpek MM, Yuen TC, Park SY, Gibbons R, Edelson DP. Using electronic health record data to develop and validate a prediction model for adverse outcomes in the wards. Crit Care Med. 2014;42(4):841-848.

37. Efron B. Logistic regression, survival analysis, and the Kaplan-Meier curve. J Am Stat Assoc. 1988;83(402):414-425.

38. Gibbons RD, Duan N, Meltzer D, et al; Institute of Medicine Committee. Waiting for organ transplantation: results of an analysis by an Institute of Medicine Committee. Biostatistics. 2003;4(2):207-222.

39. Singer JD, Willett JB. It's about time: using discrete-time survival analysis to study duration and the timing of events. J Educ Behav Stat. 1993;18(2):155-195.

40. World Health Organization. Cancer pain relief and palliative care. Report of a WHO Expert Committee. World Health Organ Tech Rep Ser. 1990;804:1-75.

41. Bailey TC, Chen Y, Mao Y, et al. A trial of a real-time alert for clinical deterioration in patients hospitalized on general medical wards. J Hosp Med. 2013;8(5):236-242.

42. Liu V, Kipnis P, Rizk NW, Escobar GJ. Adverse outcomes associated with delayed intensive care unit transfers in an integrated healthcare system. J Hosp Med. 2012;7(3):224-230. 